UCSD-PTH-05-17

\title{
The Runaway Quiver
}

\author{
Kenneth Intriligator ${ }^{1,2}$ and Nathan Seiberg ${ }^{2}$ \\ ${ }^{1}$ Department of Physics, University of California, San Diego, La Jolla, CA 92093 USA \\ ${ }^{2}$ School of Natural Sciences, Institute for Advanced Study, Princeton, NJ 08540 USA
}

\begin{abstract}
We point out that some recently proposed string theory realizations of dynamical supersymmetry breaking actually do not break supersymmetry in the usual desired sense. Instead, there is a runaway potential, which slides down to a supersymmetric vacuum at infinite expectation values for some fields. The runaway direction is not on a separated branch; rather, it shows up as a "tadpole" everywhere on the moduli space of field expectation values.
\end{abstract}

December 2005 


\section{Introduction}

There has been some recent interest in finding string theory realizations of dynamical supersymmetry breaking. In this short note, we point out that some recently proposed realizations [1-3] have runaway potentials, and therefore do not break supersymmetry in the usual desired sense. Our analysis here is a standard analysis of the field theory (for reviews see e.g. [4,5]), and some of our conclusions may already be known to some experts. However, encouraged by other experts, we will anyway present here our modest findings, in the hope that some members of the community might find it useful.

Theories with unstable, runaway directions in field space are generally not considered as viable models of supersymmetry breakingl. Runaway unstable modes can be regarded as a tadpoles, since they lead to violation of the static equations of motion. In looking for supersymmetry breaking (meta)stable ground states, one must always be careful about this potential pitfall.

A simple example with runaway is $S U(2)$ gauge theory with $N_{f}=1$ flavor, with dynamical superpotential for $\mathcal{M}=Q \widetilde{Q}[6]$

$$
W=\frac{\Lambda^{5}}{\mathcal{M}}
$$

For any finite $\langle\mathcal{M}\rangle$, there is no groundstate satisfying the static equations of motion. The potential sends $\langle\mathcal{M}\rangle \rightarrow \infty$, where asymptotically supersymmetry is restored.

Supersymmetry breaking can also be phrased as an inability to satisfy the chiral ring relations [7, [1], 3], but one must still check whether there is a stable, non-supersymmetric groundstate or a runaway direction. For the above example, the chiral ring is generated by $\mathcal{M}=Q \widetilde{Q}$, and the glueball chiral superfield $S \sim \operatorname{Tr} W_{\alpha} W^{\alpha}$. The classical ring relations are $S \mathcal{M}=0$ and $S^{2}=0$ [7]. They are deformed in the quantum theory to

$$
S \mathcal{M}=\Lambda^{5}, \quad S^{2}=0
$$

One way to see that is to follow the instanton calculations of [8]. The quantum relations (1.2) are incompatible for any finite $\langle\mathcal{M}\rangle$, but are asymptotically satisfied along the runaway direction $\langle S\rangle \rightarrow 0,\langle\mathcal{M}\rangle \rightarrow \infty$. One lesson from this example is that incompatible ring relations do not necessarily mean that supersymmetry is broken. Instead, there could be a runaway to a supersymmetric ground state at infinity.

1 They could, however, be useful for quintessence. 
Calculable examples of dynamical supersymmetry breaking without runaways were first presented in [9,10]. A review and survey of other models can be found e.g. in [5, 11, 12].

It was recently suggested [1]-3] that a string theory realization of dynamical supersymmetry breaking is obtained from IIB string theory, with wrapped D5 branes ("fractional branes"), on a Calabi-Yau space that is locally a complex cone over the surface $F_{1}$ (a.k.a. $\left.d P_{1}\right)$. See [13 for additional examples and discussion. The conformal gauge theory for D3 branes only on the $d P_{1}$ geometry was given in [14,15], and the non-conformal theory with added wrapped D5 branes in [16]. The $d P_{1}$ geometry does not admit an analog of the conifold deformation, which corresponds to the fact that, with wrapped D5 branes, the IR limit of the gauge theory is not simply SUSY Yang-Mills, with its gaugino condensation [16]. The suggestion of [1] is that the IR limit of the cascade exhibits dynamical supersymmetry breaking. This suggestion was entirely based on an analysis of the low-energy effective gauge theory. The supergravity dual solution of [17] is singular in the IR, and there is no presently known smoothed version to illuminate the IR physics.

Here we point out that this quiver gauge theory has a runaway unstable mode, everywhere on the moduli space (the runaway direction is not on a separated branch). There is no static vacuum where supersymmetry is broken and the equations of motion are satisfied. Much as in (1.1), the fields can always slide down to lower energy values, and asymptotically supersymmetry is restored (at infinite expectation values of some fields).

Our analysis is formulated in terms of the gauge theory. Perhaps some string theory dynamics - outside of the realm of the low-energy field theory analysis - somehow stabilizes the runaway mode in a way that breaks supersymmetry. In the context of compactification on a compact Calabi-Yau, this question hinges on whether and how a particular Kähler modulus is stabilized, as was recently discussed in [13]. This issue merits further study. Note that the (singular) supergravity solution of [17] is supersymmetric.

The runaway directions that we discuss were already noted in the analysis of [1, 2, 13]. It was suggested [1.13] that the D-term potentials of some $U(1)$ factors could be a cure. Here we stress that these $U(1)$ factors are anomalous, and hence massive. Therefore, their D-term equations should not be imposed. This is related to comments about "dynamical relaxation" that also already appear in some subsections of [2, [3]. We feel that it is worth stressing the bottom line: anomalous $U(1)$ D-terms should not be imposed in the low-energy theory, and they cannot prevent the runaway.

Finally, we should stress that our field theory analysis relies on a Lagrangian with canonical kinetic terms which are renormalized by field theoretic effects. It is common in 
$\mathcal{N}=1$ gauge/gravity duality that the moduli space metric requires non-canonical Kähler potential in the field theory on the branes. A different asymptotic behavior of the Kähler potential could change the conclusion about the runaway, though any asymptotically homogeneous Kähler potential will still yield a runaway - either to large or small field expectation values. A minimum could only come from an inhomogeneity in the Kahler potential.

The outline of this short note is as follows. In sect. 2, we review the fact that anomalous $U(1)$ gauge fields are massive, and that their D-term potentials should not be imposed [18, 19]. In sect. 3, we discuss the $S U(3 M) \times S U(2 M) \times S U(M)$ quiver gauge theory of $M$ wrapped D5 branes on the complex cone over $F_{1}$. The simplest case is $M=1$, where the gauge group is $S U(3) \times S U(2)$, and the matter content is similar to the 3-2 model of [10], but with an extra pair of $S U(2)$ doublets and a particular superpotential. Though the 3-2 model of [10] does dynamically break supersymmetry with a stable groundstate, this string inspired variant does not. This example also illustrates that added vector-like matter can ruin supersymmetry breaking, depending on what its tree-level superpotential couplings are.

\section{Comments on $S U(N)$ vs $U(N)$ in quiver gauge theories}

An issue that has been discussed by various authors is whether the worldvolume quiver gauge theory of branes at singularities is $\prod_{i} S U\left(N_{i}\right)$ or $\prod_{i} U\left(N_{i}\right)$. The additional $U(1)$ factors in the latter case include a decoupled, diagonal, overall $U(1)$ factor, under which no matter is charged. We will not be concerned with this $U(1)$ here. The remaining $U(1)$ factors have charged matter, and are hence IR free in four spacetime dimensions. When the string theory realization is via branes on a local, non-compact Calabi-Yau, the lowenergy gauge theory should thus be considered as $\prod_{i} S U\left(N_{i}\right)$, because the $U(1)$ couplings vanish in the IR. These couplings can be taken to be non-zero if the Calabi-Yau space is compact.

A distinct issue is the fact that the $U(1)$ factors of $\prod_{i} U\left(N_{i}\right)$ are often anomalous, with e.g. non-zero $\operatorname{Tr} S U\left(N_{i}\right)^{2} U\left(N_{j}\right)$ anomalies. This is generic for chiral quiver gauge theories, and a simple example is in D3 branes at a $\mathbb{C}^{3} / \mathbb{Z}_{3}$ orbifold singularity. As discussed in detail in [19], the worldvolume theory of the branes contains the necessary coupling to implement the Green-Schwarz anomaly cancellation mechanism as in [18]. The upshot is that anomalous $U(1)$ gauge fields $A_{\mu}$ are Higgsed by coupling to scalars $B$, through $\left(A_{\mu}-\partial_{\mu} B\right)^{2}$. 
Since anomalous $U(1)$ gauge fields are massive, they are not present in the low-energy effective field theory. For this reason, their D-term equations should not be imposed. Equivalently, supersymmetry pairs $B$ with a field $\phi$, which plays the role of the FI term for the anomalous $U(1)$ [18. The D-term of the anomalous $U(1)$ gauge field can then always relax to zero, by suitable expectation value $\langle\phi\rangle$, so it does not constrain the low-energy fields. This agrees with the discussion in [2], sect. 3.2.3. See [13] for a discussion in the context of compact Calabi-Yaus, where it is suggested that some other dynamics could perhaps induce an additional potential for the Kähler modulus $\phi$.

\section{The gauge theory}

The gauge theory of $M$ wrapped D5 branes on the complex cone over $F_{1}$ is

$\begin{array}{ccccccc} & S U(3 M) & S U(2 M) & S U(M) & {[S U(2)} & U(1)_{F} & \left.U(1)_{R}\right] \\ Q & \mathbf{3 M} & \overline{\mathbf{M}} & \mathbf{1} & \mathbf{1} & 1 & -1 \\ \bar{u} & \overline{\mathbf{3 M}} & \mathbf{1} & \mathbf{M} & \mathbf{2} & -1 & 0 \\ L & \mathbf{1} & \mathbf{2 M} & \overline{\mathbf{M}} & \mathbf{2} & 0 & 3 \\ L_{3} & \mathbf{1} & \mathbf{2 M} & \overline{\mathbf{M}} & \mathbf{1} & -3 & -1,\end{array}$

where $S U(3 M) \times S U(2 M) \times S U(M)$ are the gauge symmetries, and the groups in [.] are the global symmetries, with $U(1)_{R}$ an R-symmetry. There is a tree-level superpotential

$$
W_{\text {tree }}=h Q \bar{u}_{i} L_{j} \epsilon^{i j},
$$

where we here explicitly write the $S U(2)$ flavor indices $i, j=1,2$.

Note that we cannot extend $S U(3 M) \rightarrow U(3 M)$, because the additional $U(1)_{3 M}$ factor would be anomalous under both of the other two groups, e.g. $\operatorname{Tr} U(1)_{3 M} S U(2 M)^{2}=3 M$; similarly, we cannot extend $S U(2 M) \rightarrow U(2 M)$ or $S U(M) \rightarrow U(M)$, each of the additional $U(1)$ factors would be anomalous under both of the other two gauge groups.

The couplings, and their charges under various symmetries (some broken) are:

$\begin{array}{ccccccc} & U(1)_{Q} & U(1)_{\bar{u}} & U(1)_{L} & U(1)_{L_{3}} & U(1)_{F} & U(1)_{R} \\ \Lambda_{3 M}^{7 M} & 2 M & 2 M & 0 & 0 & 0 & 0 \\ \Lambda_{2 M}^{3 M} & 3 M & 0 & 2 M & M & 0 & 0 \\ \Lambda_{M}^{-3 M} & 0 & 6 M & 4 M & 2 M & -12 M & 0 \\ h & -1 & -1 & -1 & 0 & 0 & 0 .\end{array}$


The four symmetries $U(1)_{K=Q, \bar{u}, L, L_{3}}$ assign charge one to $K=Q, \bar{u}, L, L_{3}$, and zero to all other fields; the $U(1)_{F}$ and $U(1)_{R}$ charge assignments are as given in (3.1) $\left(U(1)_{F} \subset\right.$ $\left.U(1)_{Q} \times U(1)_{\bar{u}} \times U(1)_{L} \times U(1)_{L_{3}}\right)$. The $S U(M)$ group factor in (3.1) is IR free, as can be seen from the negative exponent in its instanton factor in (3.3). The $S U(2)$ and $U(1)_{R}$ global symmetries are preserved by the couplings. The symmetry $U(1)_{F}$ arises as an accidental symmetry in the IR, as it is broken only by the IR free group $S U(M)$.

Let us first consider the particular case of $M=1$, where the gauge group (3.1) is $S U(3) \times S U(2)$. The matter content of (3.1) in this case is similar to that of the 3-2 model of [10], but with an extra pair of $S U(2)$ doublets.

\subsection{The $S U(3) \times S U(2)$ theory $(M=1)$, in the classical limit.}

We initially consider the classical theory $\left(\Lambda_{2,3} \rightarrow 0\right)$ without the superpotential (3.2). In this case the three fields $L$ and $L_{3}$ can be combined into an $S U(3)$ triplet $L_{a=1,2,3}$. The gauge invariant fields are

$$
Z=\operatorname{det}_{f j} Q^{f} \bar{u}_{j}, \quad X_{i a}=Q \bar{u}_{i} L_{a}, \quad V^{a}=\frac{1}{2} L_{b} L_{c} \epsilon^{a b c},
$$

where the color indices are suppressed (except for the $S U(2)$ color index $f$ in the expression for $Z$ ), and the flavor indices are given. These fields satisfy the classical constraints

$$
Z V^{a}-\frac{1}{2} X_{i b} X_{j c} \epsilon^{a b c} \epsilon^{i j}=0
$$

The gauge group is completely broken for general expectation values of these fields, and the complex dimension of the classical moduli space of vacua is the number of fields left uneaten: $6+6+6-3-8=7$. This agrees with the description of the vacua in terms of expectation values of the 10 fields (3.4), subject to the 3 classical constraints (3.5).

Let us now consider the theory with added tree-level superpotential

$$
W_{\text {tree }}=h X_{i j} \epsilon^{i j},
$$

Since this interaction breaks the global $S U(3) \times S U(2)$ symmetry to $S U(2)$, we replace the index $a=1,2,3$ with $i=1,2$, and will explicitly write the $a=3$ component, so the fields are $Z, X_{i j}, X_{i} \equiv X_{i 3}, V^{i} \equiv L_{j} L_{3} \epsilon^{i j}$, and $V^{a=3}=\frac{1}{2} L_{i} L_{j} \epsilon^{i j} \equiv V$. The superpotential (3.6) lifts the $Z, X_{i j}$, and $X_{i}$ classical flat directions, but the $V$ and $V^{i}$ classical flat 
directions remain unlifted. These classical flat directions can be parameterized in terms of the original microscopic fields, up to gauge and flavor rotations, as

$$
L=\left(L_{1}, L_{2}\right)=\left(\begin{array}{cc}
c & 0 \\
0 & d
\end{array}\right), \quad L_{3}=\left(\begin{array}{c}
\sqrt{|d|^{2}-|c|^{2}} \\
0
\end{array}\right)
$$

which give $V=c d, V^{1}=-d \sqrt{|d|^{2}-|c|^{2}}$ and $V^{2}=0$, with the other fields vanishing. Along these unlifted flat directions, the $S U(2)$ gauge group is Higgsed, and the $Q$ and $\bar{u}_{i}$ matter fields get a mass from the superpotential (3.6).

There is thus a 3 complex dimensional classical moduli space of vacua left unlifted by (3.6). The low-energy spectrum along this classical moduli space is $S U(3)$ pure Yang-Mills, plus the 3 massless chiral superfields $V$ and $V^{i}$, in the $\mathbf{1}$ and $\mathbf{2}$ respectively of the global $S U(2)$ symmetry. Projecting the classical Kähler potential, $K_{c l}=Q^{\dagger} Q+u^{\dagger} u+L^{\dagger} L$ (all gauge and flavor indices are implicit and summed over), on the unlifted classical moduli space of $V$ and $V^{i}$ expectation values gives

$$
K_{c l}\left(V, V^{\dagger}, V^{i},\left(V^{i}\right)^{\dagger}\right)=2 \sqrt{T}, \quad T \equiv V V^{\dagger}+V^{i}\left(V^{i}\right)^{\dagger}=V^{a}\left(V^{a}\right)^{\dagger} .
$$

There is an accidental $S U(3)$ global symmetry, with the moduli re-combined into $V^{a}$ in the 3 , because the interactions from the superpotential (3.6), which had broken the global $S U(3)$ to $S U(2)$, do not affect the low-energy theory. Away from the origin of the moduli space, the $S U(3)$ invariant $T \neq 0$, and the $S U(3)$ symmetry is spontaneously broken to an $S U(2)$ subgroup. The 6 real massless moduli from $V^{a}$ can then be regarded the real modulus $T$, and the 5 Goldstone bosons from $S U(3) / S U(2)$.

\subsection{The $S U(3) \times S U(2)$ quantum theory for $W_{\text {tree }}=0$.}

Let us first consider the quantum theory, with $W_{\text {tree }}=0$, in the limit $\Lambda_{3} \gg \Lambda_{2}$, where the $S U(2)$ dynamics can be initially ignored. The $S U(3)$ gauge group has $N_{f}=2$ flavors, so the dynamically generated superpotential [6] is

$$
W_{d y n}=\frac{\Lambda_{3}^{7}}{Z} .
$$

Let us now consider the opposite limit, $\Lambda_{2} \gg \Lambda_{3}$. The $S U(2)$ gauge theory has $N_{f}=3$, so its low-energy spectrum consists of the $S U(2)$ gauge invariant composites, $V^{a}=\frac{1}{2} L_{b} L_{c} \epsilon^{a b c}, Q L_{a}$, and $Q^{2}$, with a superpotential term [20]. The $S U(3)$ gauge theory now has $N_{f}=3$ flavors of fundamentals, $Q L_{a}$, and anti-fundamentals, $Q^{2}$ and $\bar{u}_{i}$, so its 
low-energy spectrum consists of the gauge invariant fields with quantum deformed moduli space constraint [20]. In addition to the fields (3.4), this yields the following fields, which are classically zero: $Y_{a}=\frac{1}{2}\left(Q^{2}\right)\left(Q L_{a}\right), B=\frac{1}{6}\left(Q L_{a}\right)\left(Q L_{b}\right)\left(Q L_{c}\right) \epsilon^{a b c}$, with superpotential

$$
W_{d y n}=-\frac{1}{\Lambda_{2}^{3}}\left(B-Y_{a} V^{a}\right)+C\left(\frac{1}{2} Y_{a} X_{i b} X_{j c} \epsilon^{i j} \epsilon^{a b c}-Z B-\Lambda_{2}^{3} \Lambda_{3}^{7}\right)
$$

$C$ is a Lagrange multiplier. Integrating out the massive fields $V^{a}, Y_{a}, B$, and $C$, we find $Y_{a}=0, B=-\Lambda_{2}^{3} \Lambda_{3}^{7} / Z$, and we are left with the low-energy superpotential (3.9) and the constraint (3.5). It is also seen from the symmetries (3.3) that the constraints (3.5) could not have been modified by quantum effects.

\subsection{The $S U(3) \times S U(2)$ quantum theory with $W_{\text {tree }}=h X_{i j} \epsilon^{i j}$, for $h \neq 0$.}

The full superpotential is given by adding $W_{\text {tree }}$ to the dynamical superpotential (3.9), with the constraints (3.5) imposed with Lagrange multipliers

$$
W_{f u l l}=\frac{\Lambda_{3}^{7}}{Z}+h X_{i j} \epsilon^{i j}+\lambda_{i}\left(Z V^{i}+X_{j} X_{k l} \epsilon^{j k} \epsilon^{i l}\right)+\lambda\left(Z V-\frac{1}{2} X_{i j} X_{k l} \epsilon^{i k} \epsilon^{j l}\right) .
$$

This leads to runaway of the field $V$. Indeed, we can satisfy the supersymmetric equations of motion for all other fields via $Z=\left(\frac{\Lambda_{3}^{14}}{V h^{2}}\right)^{\frac{1}{3}}, X_{i j}=\epsilon_{i j}\left(\frac{V \Lambda_{3}^{7}}{h}\right)^{\frac{1}{3}}, \lambda_{i}=0, \lambda=\left(\frac{h^{4}}{V \Lambda_{3}^{7}}\right)^{\frac{1}{3}}$, $X_{i}=-\epsilon_{i j} V^{j}\left(\frac{\Lambda_{3}^{7}}{h V^{2}}\right)^{\frac{1}{3}}$. The low-energy spectrum consists of the fields $V$ and $V^{i}$, with the dynamical superpotential

$$
W_{\text {low }}=3\left(h^{2} V \Lambda_{3}^{7}\right)^{1 / 3}
$$

Using (3.1) and (3.3), this can be seen to be compatible with all the symmetries.

Here is another way to quickly reproduce the superpotential (3.12): the low-energy theory on the classical moduli space includes an unbroken $S U(3)$ Yang-Mills theory, with no light matter. The two $S U(3)$ flavors get a mass $\sim h \sqrt{V}$ from $W_{\text {tree }}$, so the scale of the low-energy $S U(3)$ Yang-Mills theory is given by the matching relation

$$
\Lambda_{3, \text { low }}^{9}=h^{2} V \Lambda_{3}^{7}
$$

The superpotential (3.12) arises from gaugino condensation in the $S U(3)$ Yang-Mills theory.

The superpotential (3.12) lifts the classical moduli $V$ and $V^{i}$, with potential

$$
V_{e f f}=K^{V V^{\dagger}}\left|h^{2} \Lambda_{3}^{7}\right|^{2 / 3}\left(V V^{\dagger}\right)^{-2 / 3}
$$


where $K^{V V^{\dagger}}$ is a component of the inverse Kähler metric, computed on the 3 complex dimensional moduli space of $V$ and $V^{i}$. In the limit of large $V$, we can use the classical Kähler potential (3.8). The quantum contributions of the $S U(2)$ gauge group become negligible, because it is broken at a high scale. The quantum contributions of the $S U(3)$ gauge group also become negligible for large $V$ or $V^{i}$, even though it remains unbroken. It is intuitively reasonable that the $S U(3)$ dynamics does not affect $K_{\text {eff }}$ for large $V, V^{i}$, as the only matter fields that couple $S U(3)$ to $L, L_{3}$ are the fields $Q$, which decouple as they become very massive. In this limit, the low-energy theory has the accidental $S U(3)$ global symmetry discussed following (3.8), and the effective Kähler potential must be of the form

$$
K_{e f f} \approx K_{c l}=2 \sqrt{T}=2 \sqrt{V V^{\dagger}+V^{i} V^{i \dagger}}
$$

i.e. we can use the classical Kähler potential (3.8) in (3.14). We conclude that

$$
V_{e f f} \approx\left|h^{2} \Lambda_{3}^{7}\right|^{2 / 3} \frac{2 V^{\dagger} V+\left(V^{i}\right)^{\dagger} V^{i}}{\sqrt{V^{\dagger} V+\left(V^{i}\right)^{\dagger} V^{i}}}\left(V V^{\dagger}\right)^{-2 / 3} .
$$

In the parameterization (3.7) of the classical moduli space, the potential (3.16) is

$$
V_{e f f} \approx\left|h^{2} \Lambda_{3}^{7}\right|^{\frac{2}{3}} \frac{|c|^{2}+|d|^{2}}{|c d|^{4 / 3}}
$$

For fixed $|V|$, the potential is minimized by $\left|V^{i}\right|=0$, and the remaining potential is

$$
V_{e f f} \approx 2\left|h^{2} \Lambda_{3}^{7}\right|^{2 / 3}\left(V V^{\dagger}\right)^{-1 / 6}
$$

There is thus a $V \rightarrow \infty$ runaway, and in that limit supersymmetry is restored.

The above runaway direction is present not only on the moduli space of the classical theory with nonzero $h$, but also on the larger classical moduli space of the theory with $h=0$. Furthermore, it is not isolated on a separated branch. This can also be seen by working in terms of the D-flat microscopic fields, writing the F-term potential as

$$
V_{e f f}=\left|\frac{\Lambda_{3}^{7}}{Z^{2}} Q \overline{u u}+h \bar{u} L\right|^{2}+\left|\frac{\Lambda_{3}^{7}}{Z^{2}} Q^{2} \bar{u}+h Q L\right|^{2}+|h Q \bar{u}|^{2}
$$

(we suppressed the color and flavor indices which are summed over). Extremizing in the fields that can have supersymmetric minima, the only light fields are the classical moduli $V$ and $V^{i}$, with low-energy potential (3.16). There are no other branches. 
To summarize, we have found that the effective potential has runaway behavior and no metastable SUSY-breaking minimum, at least in the regime of large $\langle V\rangle$, where the Kähler potential is approximately canonical. The dynamical supersymmetry breaking of the original $S U(3) \times S U(2)$ model of [9] would be recovered if we could add a mass term $W_{\text {mass }}=m L_{2} L_{3}$ to the tree-level superpotential. But, in the context of this brane system, such a mass term is forbidden by the $S U(2)$ global symmetry; this global symmetry is ensured by the isometry of the space $d P_{1}$. There is a mass term which respects the $S U(2)$ global symmetry, $W_{m a s s^{\prime}}=-\frac{m}{2} L_{i} L_{j} \epsilon^{i j}=-m V$, but adding that mass term leads to a supersymmetric ground state with $\langle V\rangle=h \Lambda_{3}^{7 / 2} / m^{3 / 2}$. As $m \rightarrow 0$ this states moves to infinity.

\subsection{The generalization to $M>1$ wrapped branes.}

The gauge theory (3.1) with superpotential $W_{\text {tree }}(3.2)$ has runaway direction:

$$
\left(L_{1}, L_{2}\right)=\left(\begin{array}{cc}
c \mathbf{1}_{M \times M} & \mathbf{0}_{M \times M} \\
\mathbf{0}_{M \times M} & c \mathbf{1}_{M \times M}
\end{array}\right),
$$

with $\mathbf{1}_{M \times M}$ an $M \times M$ unit matrix, and $\mathbf{0}_{M \times M}$ a vanishing $M \times M$ matrix. The gauge invariant light field along this direction is $V \equiv \operatorname{det}_{2 M \times 2 M}\left(L_{1}, L_{2}\right)=c^{2 M}$, and the group is Higgsed as $S U(3 M) \times S U(2 M) \times S U(M) \rightarrow S U(3 M) \times S U(M)^{\prime}$, where $S U(M)^{\prime}$ is a diagonal subgroup of $S U(2 M) \times S U(M)$. The $S U(3 M)$ group has no massless flavors, as the fields $Q$ and $\bar{u}_{i}$ get a mass along this direction, because of $W_{\text {tree }}$ (3.2). The $S U\left(M^{\prime}\right)$ have two massless adjoints, coming from the fields $L_{3}$. The fields $L_{3}$ also yield two massless singlets, corresponding to the gauge invariants $V_{i}=\operatorname{det}_{2 M \times 2 M}\left(L_{i}, L_{3}\right)$.

The dynamical scales of the low-energy theory are given by the matching relations

$$
\Lambda_{3 M, l o w}^{9 M}=h^{2 M} V \Lambda_{3 M}^{7 M}, \quad \Lambda_{M, l o w}^{M}=\left(\Lambda_{2 M}^{3 M}\right)^{2} \Lambda_{M}^{-3 M} / V
$$

where the exponents in the latter scale relation are because of the index of the embedding of $S U(M)^{\prime} \subset S U(2 M) \times S U(M)$. The effective superpotential along the runaway direction comes from gaugino condensation in the low-energy $S U(3 M)$ Yang-Mills theory (the $S U(M)^{\prime}$ theory has too much massless matter to dynamically generate a superpotential):

$$
W_{\text {low }}=3 M\left(h^{2 M} V \Lambda_{3 M}^{7 M}\right)^{1 / 3 M} .
$$

The canonical Kähler potential for $V$ is $K_{\text {can }} \sim\left(V V^{\dagger}\right)^{1 / 2 M}$, so the potential for large $V$ is $V_{\text {eff }} \sim\left|\left(h^{2 M} \Lambda^{7 M}\right)^{2} V^{-1}\right|^{1 / 3 M}$, which again has a runaway $\langle V\rangle \rightarrow \infty$. 


\section{Acknowledgments:}

We would like to thank I. Bena, I. Klebanov, J. Maldacena and especially D. Berenstein, S. Kachru, D. Shih for useful discussions. The research of NS is supported in part by DOE grant DE-FG02-90ER40542. The research of KI is supported in part by UCSD grant DOE-FG03-97ER40546 and by the IAS Einstein Fund. KI would like to thank the IAS for their hospitality and support on his sabbatical visit. NS would like to thank the

hospitality of The Institute for Advanced Studies of The Hebrew University in Jerusalem where this work was completed. 


\section{References}

[1] D. Berenstein, C. P. Herzog, P. Ouyang and S. Pinansky, "Supersymmetry breaking from a Calabi-Yau singularity," JHEP 0509, 084 (2005) arXiv:hep-th/0505029.

[2] S. Franco, A. Hanany, F. Saad and A. M. Uranga, "Fractional branes and dynamical supersymmetry breaking," arXiv:hep-th/0505040.

[3] M. Bertolini, F. Bigazzi and A. L. Cotrone, "Supersymmetry breaking at the end of a cascade of Seiberg dualities," Phys. Rev. D 72, 061902 (2005) arXiv:hep-th/0505055.

[4] K. A. Intriligator and N. Seiberg, "Lectures on supersymmetric gauge theories and electric-magnetic duality," Nucl. Phys. Proc. Suppl. 45BC, 1 (1996) arXiv:hepth/9509066].

[5] J. Terning, "Non-perturbative supersymmetry," arXiv:hep-th/0306119.

[6] I. Affleck, M. Dine and N. Seiberg, "Dynamical Supersymmetry Breaking In Supersymmetric QCD," Nucl. Phys. B 241, 493 (1984).

[7] N. Seiberg and E.Witten, unpublished.

[8] D. Amati, K. Konishi, Y. Meurice, G. C. Rossi and G. Veneziano, "Nonperturbative Aspects In Supersymmetric Gauge Theories," Phys. Rept. 162, 169 (1988).

[9] I. Affleck, M. Dine and N. Seiberg, "Calculable Nonperturbative Supersymmetry Breaking," Phys. Rev. Lett. 52, 1677 (1984).

[10] I. Affleck, M. Dine and N. Seiberg, "Dynamical Supersymmetry Breaking In FourDimensions And Its Phenomenological Implications," Nucl. Phys. B 256, 557 (1985).

[11] E. Poppitz and S. P. Trivedi, "Dynamical supersymmetry breaking," Ann. Rev. Nucl. Part. Sci. 48, 307 (1998) arXiv:hep-th/9803107.

[12] Y. Shadmi and Y. Shirman, "Dynamical supersymmetry breaking," Rev. Mod. Phys. 72, 25 (2000) arXiv:hep-th/9907225.

[13] D. E. Diaconescu, B. Florea, S. Kachru and P. Svrcek, "Gauge - Mediated Supersymmetry Breaking in String Compactifications," arXiv:hep-th/0512170.

[14] C. Beasley, Senior Thesis, Duke University

[15] B. Feng, A. Hanany and Y. H. He, "D-brane gauge theories from toric singularities and toric duality," Nucl. Phys. B 595, 165 (2001) arXiv:hep-th/0003085.

[16] F. Cachazo, B. Fiol, K. A. Intriligator, S. Katz and C. Vafa, "A geometric unification of dualities," Nucl. Phys. B 628, 3 (2002) arXiv:hep-th/0110028.

[17] C. P. Herzog, Q. J. Ejaz and I. R. Klebanov, "Cascading RG flows from new SasakiEinstein manifolds," JHEP 0502, 009 (2005) arXiv:hep-th/0412193.

[18] M. Dine, N. Seiberg and E. Witten, "Fayet-Iliopoulos Terms In String Theory," Nucl. Phys. B 289, 589 (1987).

[19] M. R. Douglas and G. W. Moore, "D-branes, Quivers, and ALE Instantons," arXiv:hep-th/9603167.

[20] N. Seiberg, "Exact results on the space of vacua of four-dimensional SUSY gauge theories," Phys. Rev. D 49, 6857 (1994) arXiv:hep-th/9402044]. 